\title{
Quiste pilonidal del ombligo: presentación de dos casos
}

\author{
Juan Hepp K. ${ }^{1,2}$, Horacio Ríos R. ${ }^{1,2}$, Juan Hepp V. ${ }^{3}$ y Paulina Fuenzalida S. ${ }^{3}$
}

'Clínica Alemana de Santiago. Santiago, Chile. 2Facultad de Medicina Clínica Alemana Universidad de Desarrollo. Santiago, Chile. ${ }^{3}$ Facultad de Medicina Universidad de los Andes. Santiago, Chile.

Recibido 2020-06-27 y aceptado 2020-07-05

Correspondencia a: Dr. Juan Hepp K. jhepp@alemana.d

\section{Umbilical pilonidal sinus: presentation of two cases}

Introduction: Umbilical pilonidal sinus (UPS) is a rare condition and is therefore not detected early. The aim of this document is to discuss this pathology. Case report: We present two patients referred due to an inflammatory process of the navel, with the presence of a malodor. The first patient, after two months of unsuccessful local treatment, was intervened by a resection of the umbilical lesion, verifying hair content in the sinus. In the second patient the diagnosis was evident during anamnesis and confirmed with the physical examination. In both cases, a partial resection of the umbilicus was performed, including the pilonidal sinus. Biopsy confirmed the diagnosis. The postoperative result has been satisfactory, without relapse. Discussion and Conclusion: There are not much literature related to UPS. Conservative treatment or surgical resection of the sinus is proposed. In our limited experience we proceeded to a surgical resection of the lesion and recommend eliminating the hairs of the periumbilical region. We cannot rule out the conservative management option in future cases, before proposing surgery.

Key words: pilonidal sinus; pilonidal cyst; sinus.

\section{Resumen}

Introducción: El quiste pilonidal del ombligo (QPO) es una entidad muy infrecuente y por eso no es reconocida precozmente. El objetivo de este manuscrito es dar a conocer esta patología. Reporte de casos: Presentamos dos pacientes referidos por un proceso inflamatorio del ombligo, con descarga de mal olor. El primer paciente, luego de dos meses de tratamiento local sin resolución, es intervenido resecando la lesión umbilical, comprobando en ese momento la existencia de contenido piloso en el quiste. En el segundo paciente se plantea el diagnóstico durante la anamnesis y se confirma con el examen físico. En ambos casos se efectuó una resección parcial del ombligo incluyendo la lesión pilonidal. La biopsia confirmó el diagnóstico. El resultado posoperatorio ha sido satisfactorio y sin recaídas. Discusión y Conclusión: Hay escasa literatura relacionada con el QPO. Se proponen el tratamiento conservador y la opción de resección quirúrgica del quiste. En nuestra limitada experiencia se procedió a resecar la lesión y recomendar la depilación de la región periumbilical. No podemos descartar la alternativa de manejo conservador en futuros casos, antes de proponer la cirugía.

Palabras clave: quiste pilonidal; quiste pilonidal umbilical; pilonidal.

\section{Introducción}

El quiste pilonidal del ombligo (QPO) es una entidad clínica muy inhabitual y poco conocida, a diferencia de la enfermedad pilonidal sacrococcígea que se observa con frecuencia en nuestro medio. La descripción del QPO y la nominación de "Pilonidal sinus of the umbilicus" es atribuida a David Patey en 1956 ${ }^{1}$. Posteriormente, hay diversas publicaciones de uno o varios casos clínicos, recolección de casos de diversas publicaciones y últimamente un estudio multicéntrico, prospectivo y randomizado con 84 casos en Turquía, que han permitido caracterizar esta condición y su tratamiento $^{2}$. Tanto el QPO y el quiste sacrococcígeo se asocian con el sobrepeso, la compresión de la zona y la carga pilonidal en su vecindad, factores que contribuirían a su generación ${ }^{3}$. El objetivo de este manuscrito es dar a conocer esta entidad clínica, lo que permitirá reconocerla precozmente y evitar sucesivas consultas a diferentes especialistas o tratamientos inadecuados. 


\section{Reporte de casos clínicos}

\section{Caso 1}

Hombre de 54 años, sin enfermedades concomitantes, operado de várices y hernia inguinal. Nota desde mediados de junio de 2019 descarga de líquido de mal olor intermitente del ombligo. Al examen se observa la piel del abdomen con abundantes vellos, el ombligo profundo, en cuyo fondo y hacia lateral tiene una lesión solevantada, violácea, indurada, discretamente sensible, con secreción de mal olor. Tratado con aseo local y antibióticos se logra reducción parcial del proceso, pero con reaparición posterior. En ecografía de la pared abdominal se describe en relación al ombligo una pequeña hernia, con anillo de $5 \mathrm{~mm}$ y contenido adiposo de $8 \mathrm{~mm}$. Exámenes de hemograma, perfil bioquímico, orina estaban en rengos normales.

Luego de 10 semanas de evolución se efectuó exploración quirúrgica del ombligo, comprobando una lesión quística con abundantes pelos en su interior, se resecó $1 / 3$ de la piel del ombligo incluyendo la lesión quística y sutura de una mínima hernia umbilical, reconstituyendo luego la conformación del ombligo (Figuras 1 y 2). La evolución posterior fue muy buena, con adecuada estética umbilical y sin contratiempos. Se recomendó rasurado de la vecindad del ombligo. La biopsia fue informada como "compatible con quiste pilonidal, fibrosado, con reacción inflamatoria crónica y aguda, sin atipias". En control a los 6 meses de la cirugía se encuentra sin contratiempos.

\section{Caso 2}

Hombre de 71 años, portador de cuadros respiratorios reactivos, operado de pólipos laríngeos, resección transuretral de próstata y colecistectomía por laparoscopia, con incisión en el borde superior del ombligo. Consulta a fines de enero de 2020 y refiere 3 meses se supuración intermitente del ombligo, con un solevantamiento en su interior. Había sido visto por otros especialistas previamente, se le recomendó aseo local y ungüento tópico sin respuesta clínica suficiente. Con la experiencia obtenida meses antes con el paciente descrito en el caso 1, se le planteó la posibilidad que sea un QPO. Al realizar el examen físico del abdomen se observa abundante pilosidad cutánea, el ombligo grueso y profundo con una cicatriz en el borde superior del ombligo (cirugía laparoscópica) de caracteres normales. En el borde inferior del ombligo había un proceso inflamatorio local, líquido de mal olor, con un bulto del cual, con una pinza, es posible extraer un manojo de pelos de 10 a $15 \mathrm{~mm}$ de largo.
Diez días más tarde se efectuó la extirpación quirúrgica del QPO con reconstrucción del ombligo. Su evolución no tuvo contratiempos, con buena cicatrización local. Se le recomendó el rasurado de la zona. La biopsia fue informada como "hallazgos morfológicos compatibles con quiste pilonidal roto e inflamado".

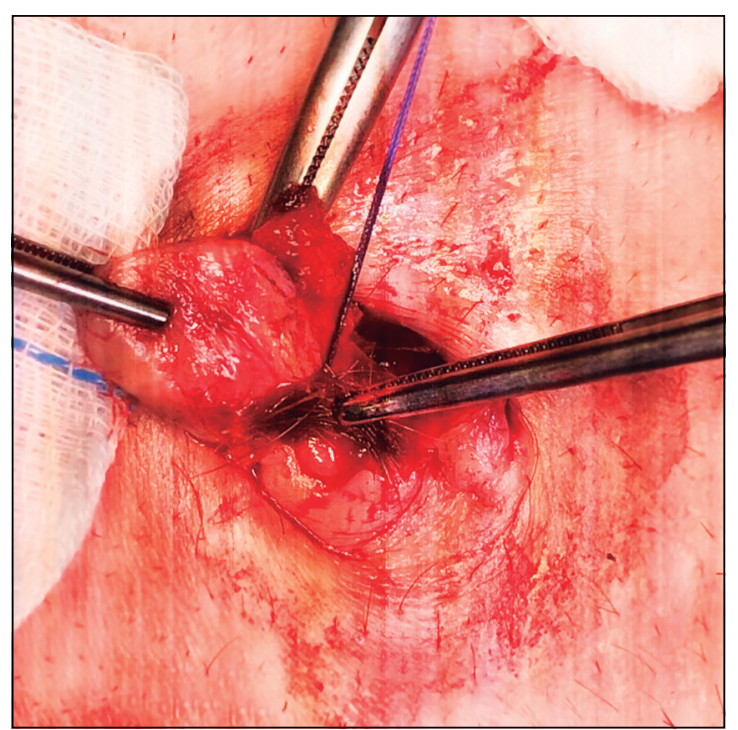

Figura 1. Quiste pilonidal del ombligo. Piel abdominal con abundantes pelos y lesión inflamatoria intraumbilical.

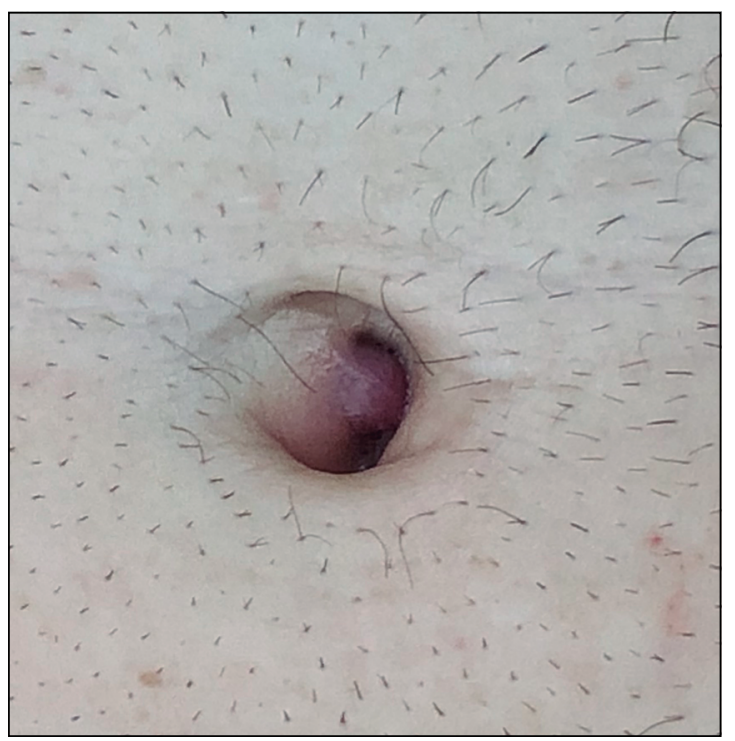

Figura 2. Quiste pilonidal del ombligo, resección quirúrgica de la lesión. Se observa pinza que extrae un penacho de pelos del quiste. 


\section{Discusión y revisión de la literatura}

No es raro que casos inhabituales se presenten en una serie clínica y, luego de aprender de la primera experiencia, los casos siguientes son de diagnóstico y tratamiento efectivo y satisfactorio. Los dos casos descritos tienen una gran similitud en su presentación clínica, características de los pacientes y buen resultado con la resección quirúrgica de la lesión. Ambos pacientes con abundantes pelos en el abdomen y ombligo profundo, como se ha descrito en la literatura quirúrgica ${ }^{3}$.

$\mathrm{Clery}^{4}$, cita que el primer caso de QPO fue planteado por Jackson en la discusión de un caso en 1854 y que, posteriormente, existen otras referencias de quistes pilosos en diferentes partes del cuerpo, incluyendo el ombligo.

Fue David Patey quien describió en 1956 un caso de QPO y lo denominó Pilonidal sinus of the umbilicus $^{1}$. Plantea que esta es una condición adquirida, en personas con piel abdominal pilosa, y que la migración de los pelos al ombligo puede ser por succión desde el ombligo, crecimiento de los pelos hacia el ombligo o por fricción entre la ropa y la pared abdominal, migrando trozos de pelos al interior del ombligo. Se inclina por la teoría de la fricción, en que los pelos generan un traumatismo en la profundidad de la piel del ombligo, su perforación y acumulación en penachos dentro de una cavidad o quiste. Con posterioridad hay diversas publicaciones de casos aislados o pequeños grupos de pacientes similares, en los cuales se plantea el tratamiento conservador o su resección, con diferente énfasis.

Eryilmaz y cols ${ }^{3}$, publican en 2005 una serie de 26 casos de QPO, de un centro en Turquía, señalando que son factores predisponentes el género masculino, jóvenes con piel pilosa, ombligo profundo, sobrepeso e hiperhidrosis. Recomiendan un tratamiento conservador con curetaje del quiste y extracción de los pelos, curaciones y manejo del granuloma con nitrato de plata. En esta serie, 23 pacientes curaron con estas medidas y 3 requirieron resección quirúrgica del quiste, por fracaso del tratamiento conservador ( 2 casos) o por asociación con hernia umbilical (1 caso). Recomiendan el manejo conservador como primera instancia.

En el 2008, Fazeli y cols ${ }^{5}$ reportan una serie desde Irán, reuniendo 45 pacientes con QPO que fueron tratados mediante resección del ombligo y su reconstrucción posterior. En 6 pacientes había un quiste pilonidal sacrococcígeo sincrónico. El resultado de la cirugía en el QPO fue muy satisfactorio, con $9 \%$ de infección de herida operatoria, sin recidivas en un seguimiento entre 3 y 62 meses. Recomiendan un criterio terapéutico resectivo.

Kareem comunica en $2013^{6}$, la experiencia en Iraq de 134 pacientes con QPO, con seguimiento de 105 de ellos tratados prioritariamente de forma conservadora (76\%), removiendo los pelos del quiste, desbridando la cavidad con anestesia local, con curaciones posteriores y uso de antibióticos. Solo 3 pacientes requirieron cirugía resectiva.

En 2016 Kaplan y cols ${ }^{2}$, publicaron un estudio multicéntrico en Turquía, prospectivo y randomizado, en 84 pacientes, comparando el manejo conservador con la cirugía. A los 2 años de seguimiento mejora el $68 \%$ de los pacientes que recibieron tratamiento conservador y el $100 \%$ de los tratados con cirugía resectiva. Se observó que el tratamiento conservador requiere de un tiempo más prolongado, tiene mayor recurrencia y menor tasa de curación en 2 años plazo.

\section{Conclusiones}

El conocimiento de la existencia del QPO nos permite enfrentar oportunamente un tratamiento con un diagnóstico adecuado, pudiendo optar entre un manejo conservador con aseo adecuado de la lesión, curaciones, empleo de antibiótico y seguimiento, o bien con la resección quirúrgica de la lesión, que permite una resolución más rápida y con menos riesgo de fracaso. En los dos casos relatados por nosotros optamos por esta última opción, con un resultado muy satisfactorio. La opción de un manejo conservador es factible y requiere de un tratamiento más prolongado, en manos de un cirujano que conozca el tema y un paciente bien informado.

\section{Responsabilidades éticas}

Protección de personas y animales. Los autores declaran que para esta investigación no se han realizado experimentos en seres humanos ni en animales.

Confidencialidad de los datos. Los autores declaran que en este artículo no aparecen datos de pacientes.

Conflictos de interés: no hay. 


\section{Bibliografía}

1. Patey D, Williams E. Pilonidal sinus of the umbilicus. Lancet 1956;271:281-2.

2. Kaplan M, Ozcan O, Kaplan FC,

Yalcin HC, Salman B. Conservative vs

Surgical Interventions for Umbilical

Pilonidal Sinus: A Multicenter,

Double-Blind, Prospective, Randomized
Clinical Trial. J Am Coll Surg. 2016;222:878-89.

3. Eryilmaz R, Sahin M, Okan O, Alimoglu O, Somay A. Umbilical pilonidal sinus disease: predisposing factors and treatment. World J Surg. 2005;29:1158-60.

4. Clery AP, Clery AB. Pilonidal disease of the umbilicus. Br J Surg. 1963;50:666-8.

5. Fazeli MS, Lebaschi AH, Adel MG,
Kazemeini AR. Evaluation of the Outcome of Complete Sinus Excision with Reconstruction of the Umbilicus in Patients with Umbilical Pilonidal Sinus. World J Surg. 2008;32:2305-8.

6. Kareem T. Outcomes of Conservative Treatment of 134 Cases of Umbilical Pilonidal Sinus. World J Surg. 2013;37:313-7. 